IOSR Journal of Pharmacy

e-ISSN: 2250-3013, p-ISSN: 2319-4219, www.iosrphr.org

Volume 2 Issue 6 || $\mid$ Nov-Dec. 2012 || | PP.01-08

\title{
Developing Cancer Immunotherapies by the manipulation of Immune Checkpoints
}

\author{
Kondadasula Sri Yogesh \\ Indian Institute of Science Education and Research Bhopal
}

\begin{abstract}
A tumor mass consists of cells that have undergone a large number of mutations, these mutations might provide a diverse set of antigens that are capable of producing an immune response. Therefore cancer cells need to suppress the immune system to form a sustainable tumor mass. Hence, tumors co-opt certain immune checkpoint pathways to suppress the immune response in the tumor microenvironment. Immune checkpoints are the inhibitory pathways used by the immune system to maintain self-tolerance and modulate the immune response in peripheral tissues to minimize the collateral tissue damage, to avoid autoimmune disorders. Most of the immune checkpoints are initiated by ligand-receptor interactions; therefore the blockade of either the ligand or the receptor with the help of the specific antibodies can suppress the activity of these immune checkpoints. Hence one of the most promising approaches to activate antitumor immunity is by the blockade of immune checkpoints. A Cytotoxic T lymphocyte associated antigen 4 (CTLA4) antibody, named Ipilimumab, was the first such immunotherapeutic to achieve US FDA approval. Preliminary clinical findings with blockers of additional immune checkpoint proteins, such as Programmed cell death protein 1 (PD1), indicate broad and diverse opportunities to enhance antitumor immunity with the potential to produce durable clinical responses.
\end{abstract}

Keywords-APCs, Immune Checkpoints, Objective responses, response rates, TCR, $T_{\text {Reg }}$ cells.

\section{INTRODUCTION}

Conventionally cancers are treated by three methods, which are, Surgery, Chemotherapy and Radiation therapy. As we know that the current conventional therapies are incapable of delivering a solid anti-tumor therapeutic without any pronounced side effects, we are hence in search of a new therapeutic that can eliminate tumors and also have very little to no side effects. Immunotherapy, which basically means to manipulate the immune system in order to obtain a suitable immune response to eliminate the tumor, is currently being developed to be used as a standalone therapeutic in many different types of cancers. Tumor cells are formed from many different types of mutations; these mutations provide a diverse set of antigens that are capable of producing an immune response. So the question comes here, why is the immune system not able to eliminate the tumors all by itself? The question can be answered using recent observations that in the tumor micro environment the immune checkpoint receptors and ligands are over expressed and hence suppress the immune response against the tumor cells. Immune checkpoint pathways are those inhibitory pathways used by the immune system to regulate the immune response in peripheral tissues in order to reduce the collateral tissue damage. As most of these immune checkpoint pathways are activated by ligand receptor interactions, blockade of either the ligand or the receptor using specific monoclonal antibodies can stop these immune checkpoint pathways from getting activated, hence we can then see a durable immune response against the tumor cells. In this review, few of the immune checkpoint pathways will be discussed along with the clinical application and status of few antibodies that are being used to block the immune checkpoint pathways.

\section{CTLA4 IMMUNE CHECKPOINT}

$\mathrm{T}$ cell co-stimulatory receptor, $\mathrm{CD} 28$, does not affect $\mathrm{T}$ cell activation unless the $\mathrm{T}$ cell receptor (TCR) is first engaged by its particular antigen. After the antigen is recognized, CD28 signalling amplifies the TCR signalling to activate the T cells. Cytotoxic T lymphocyte associated antigen 4 (CTLA4), also present on T cells, primarily regulates the amplitude of the early stages of T cell activation. CTLA4 shares the same ligands with CD28, CD80 (also known as B7.1) and CD86 (also known as B7.2) [1 to5]. CTLA4 having a higher overall affinity towards these ligands competitively inhibits CD28. Not only does CTLA4 competitively inhibit CD28 but it also has a signalling mechanism that involves the activation of protein phosphatases such as SHP2 (PTPN11) and PP2A that counteract the kinase signals induced by the TCR and CD28 [6 to 12]. 

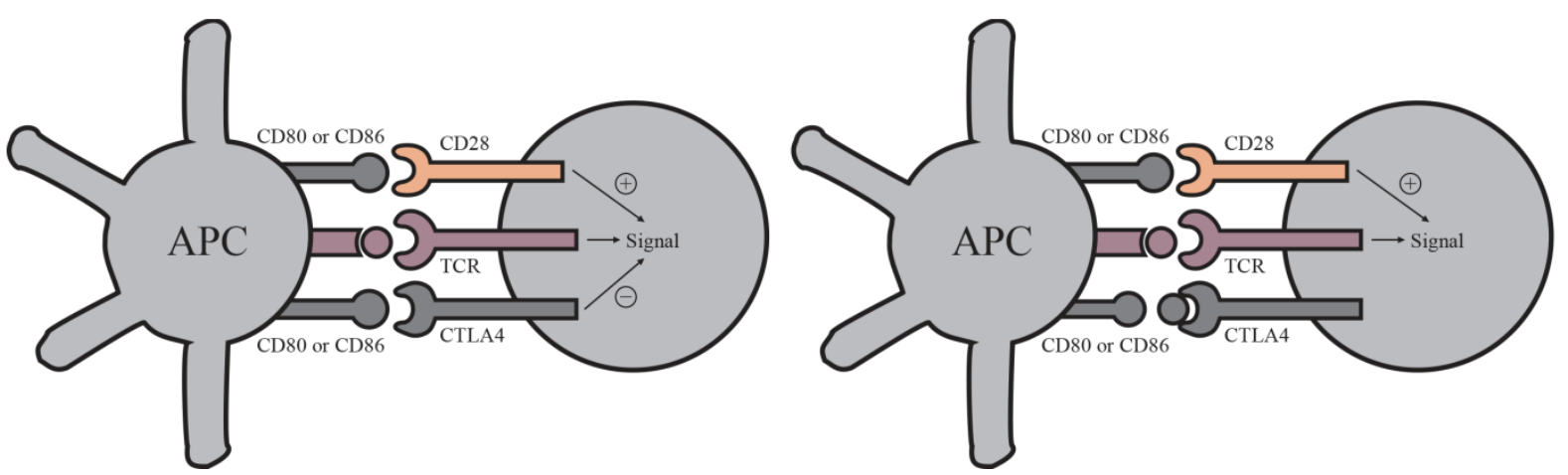

Figure 1. The left portion of the above figure shows how CTLA4 competitively inhibits CD28 (T cell co-stimulatory receptor) in binding to $\mathrm{CD80}$ or $\mathrm{CD86}$ and how the phosphatases counteract the kinase signals produced by the TCR (T cell Receptor) and CD28. The right portion of the figure signifies the CTLA4 blockade by an antibody.

CTLA4 receptors are also known for the removal of CD80 and CD86 ligands from the surface of the Antigen Presenting Cells (APC) [13], to down modulate the Helper $\mathrm{T}$ cells and to amplify the immunosuppressive function of the Regulatory $T$ cells $\left(T_{\text {reg }}\right)$ [14 to 16]. CTLA4 knockout mice were found out to be lethally autoimmune and also the T-cells were hyperactivated $[17,18]$, therefore the central role of CTLA4 is to keep the T-cell activation in check. $\mathrm{T}_{\text {reg }}$ cells also express CTLA4, but the mechanism by which CTLA4 enhances the immunosuppressive function of the $\mathrm{T}_{\text {reg }}$ cells is currently not known. Therefore, CTLA4 blockade could enhance the effector $\mathrm{T}$ cell activity and also reduce the $\mathrm{T}_{\text {reg }}$ cell dependent immunosuppression.

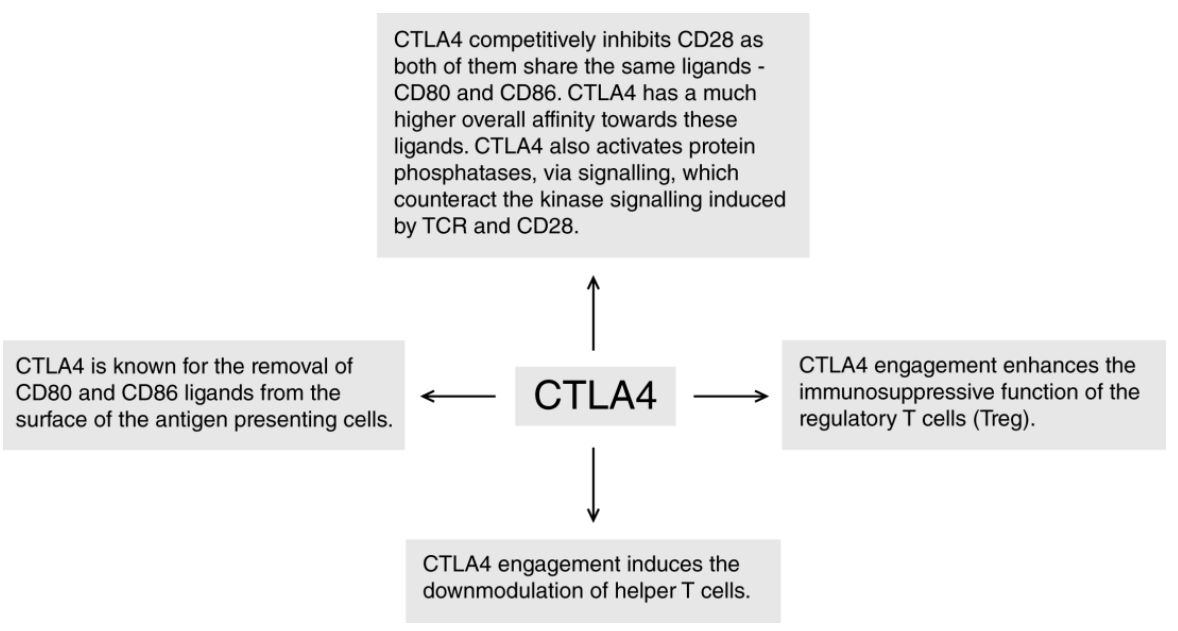

Figure 2. The above diagram represents the working of a CTLA4 immune checkpoint

\section{CLINICAL APPLICATION OF CTLA4 BLOCKADE}

Preclinical findings encouraged the production of two fully humanized CTLA4 antibodies, Ipilimumab and Tremelimumab, which began testing in 2000. After the phase III clinical trials of Tremelimumab were concluded, it was noted that tremelimumab showed no survival benefits with dose and schedule relative to Dacarbazine (DTIC) [19], which is a standard chemotherapeutic drug used in the treatment of melanoma. Even though the response rates and immune toxicity profiles of both the antibodies in Phase II trials were similar, Ipilimumab was more carefully evaluated at different doses and schedules. A three arm clinical trial [20], that lead to the US FDA approval of ipilimumab is shown in Fig.3. 


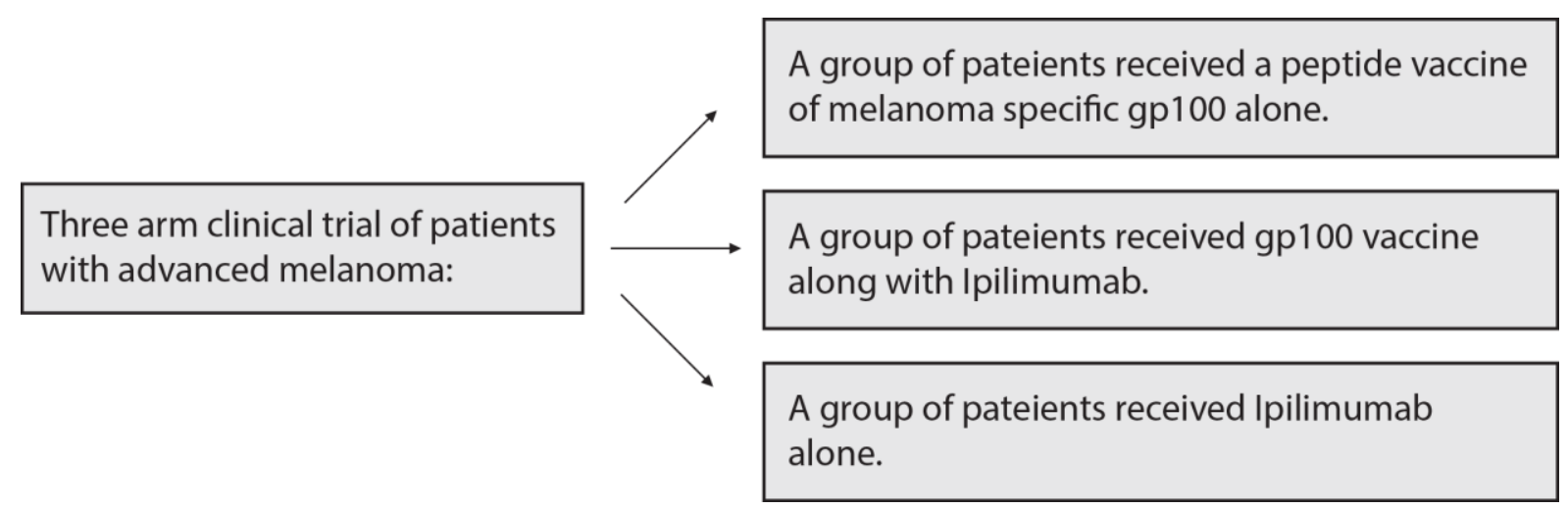

Figure 3. The above figure shows the three arm clinical trial conducted in patients suffering from advanced melanoma. A group of patients received a peptide vaccine, gp100, which stimulates the immune system against the tumor. The next group received gp100 along with ipilimumab and the last group received ipilimumab as a single therapeutic agent alone.

In the above randomized three arm clinical trial of patients with advanced melanoma, shown in Fig. 3, patients treated with ipilimumab with or without the gp100 vaccine showed a mean 3.5 months survival benefit. Dacarbazine was approved by the US FDA on the basis of response rates but has not been shown to provide a survival benefit in patients with melanoma. Therefore, as Ipilimumab was the very first therapy to show a survival benefit, it was approved by the US FDA for the treatment of advanced melanoma in 2010. More impressive than the mean survival benefit was the effect of Ipilimumab on long term survival, that is, the proportion of long term survivors was higher than the proportion of objective responders [20]. Hence this finding of ongoing responses and survival long after completion of a relatively short course of therapy (four doses of $10 \mathrm{mg}$ per $\mathrm{kg}$ over 3 months) support the concept that immune based therapies might reeducate the immune system to keep tumors in check after the completion of therapeutic intervention [21].

\section{PD1 IMMUNE CHECKPOINT}

The major role of PD1 is to limit the activity of $\mathrm{T}$ cells in the peripheral tissues at the time of an inflammatory response to infection and to restrict autoimmunity [22 to 28]. Within the tumor microenvironment, this translates into a major immune resistance mechanism [29 to 31]. PD1 expression is induced when the T cells are activated. When engaged by one of it's ligands, PDL1(B7-H1) and PDL2(B7-DC) [32 to 34], PD1 through help of the SHP2 phosphatase inhibits kinases that are involved in the T cell activation [32], it is shown in Fig.4. PD1 engagement also inhibits the TCR stop signal which could modify the duration of T cell-APC or T cell-target cell contact [35]. Similar to CTLA4, PD1 is highly expressed on $\mathrm{T}_{\text {reg }}$ cells, where it may enhance their proliferation in the presence of a ligand [36]. PD1 is more broadly expressed than CTLA4, as it is induced on other activated non T cell subsets, including B cells and the Natural Killer (NK) cells [37, 38]. Therefore, although PD1 blockade is typically viewed as enhancing the activity of effector $\mathrm{T}$ cells in the tumor microenvironment, it probably enhances NK cell activity and may also enhance the antibody production either indirectly or through directeffects on PD1+ B cells [39].

\subsection{Anamolous Behaviour of CD80}

CD80 which acts as a ligand for the T cell co-stimulatory receptor (CD28) and CTLA4 was recently found to function as a receptor when engaged to PDL1, delivering inhibitory signals and reducing the effector T cell functions $[40,41]$.

\subsection{Blockade of PDL1}

On the surface of solid tumor cells, the major ligand expressed is PDL1. Based on the known interactions of PD1 ligands, it is theoretically possible that a PD1 antibody would have a distinct biological function compared to a PDL1 antibody. A PD1 antibody would block the interaction of PD1 with both PDL1 and PDL2, but it cannot block the interactions of PDL1 and CD80. In contrast, the PDL1 antibody would block the PDL1 interaction with PD1 and CD80, but cannot block the interaction of PD1 and PDL2. Thus, depending on which type of interaction dominates in a particular cancer, antibodies for PD1 or PDL1 may be used. There is also a theoretical probability of PD1 and PDL1 antibodies to show synergistic effects, when used together. 


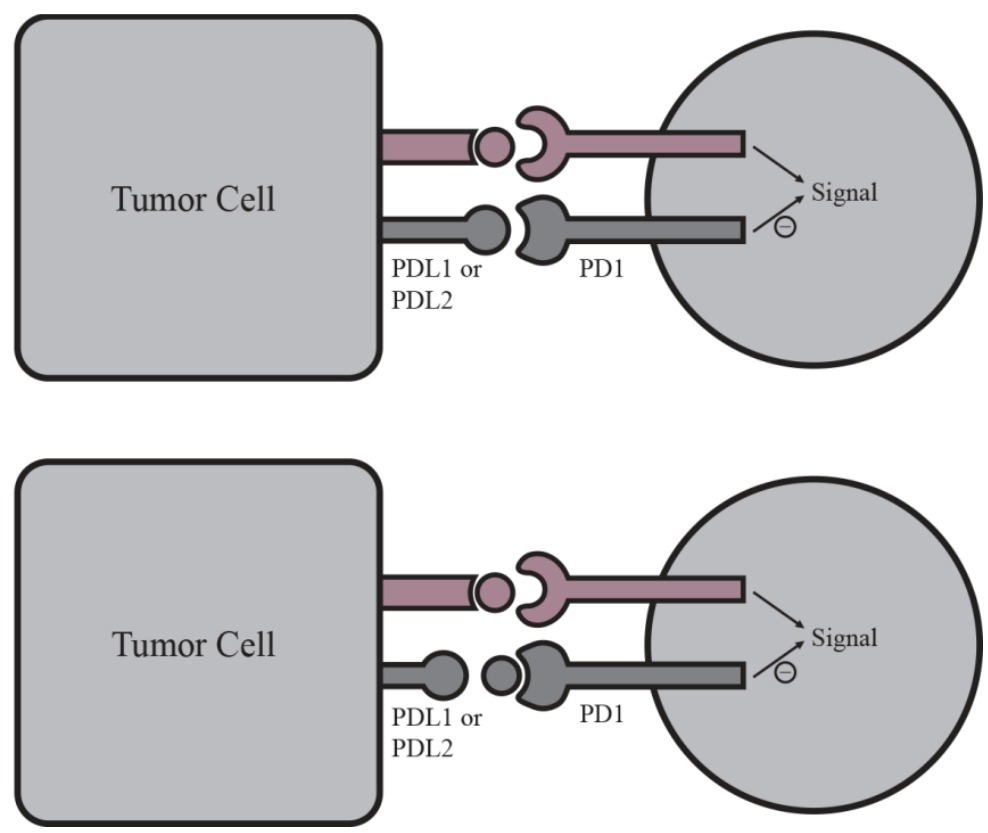

Figure 4. The upper portion of the above figure shows how the production of phosphatases, which are produced via a signaling mechanism when the PD1 receptor is engaged with it's ligand, counteract the kinase signaling of the effector $T$ cell. The lower portion of the figure shows the enhancement of the effector $T$ cell signals when the PD1 receptor is blocked by an antibody that is optimized to specifically bind to the PD1 receptor.

\section{CLINICAL APPLICATION OF PD1 BLOCKADE}

The clinical experience with PD1 antibodies is currently much less extensive than with CTLA4 antibodies, but the initial results do look extremely promising. The initial results from a clinical trial, extending the treatment with anti-PD1 to two years, objective responses were observed in 16 out of 39 patients with advanced melanoma, and an additional 14 patients achieved either a partial response or disease stabilization [42]. Similar response rates have been observed in renal cancer [43], and there is an ongoing evaluation of antiPD1 in lung cancer. As the frequency of immune related toxicities from anti-PD1 therapy seem to be less than in anti-CTLA4 therapy, and with the promising results of the initial clinical trials, we can conclude that anti PD1 immunotherapy could indeed be a potential immunotherapy that can be standardized for the treatment of multiple cancers.

\section{COMBINATION THERAPIES}

Preclinical models validate the dramatic synergy between tumor vaccines and blockade of the immune checkpoint receptors such as CTLA4 and PD1. Given below, in Fig.5, is a diagramatic expalantion of the working of combination immunotherapies. In some instances when the tumor is poorly immunogenic, then the anti-tumor immune response, enhanced by the immune checkpoint blockers, would not be effective enough to eliminate the entire tumor. Therefore anti tumor immunity enhancing vaccines must be administered before the blockade of the immune checkpoints inorder to see an effective anti tumor immune response.

So, the future immunotherapies must be developed by keeping in mind the synergy between immune enhancing vaccines and immune checkpoint blockers. 

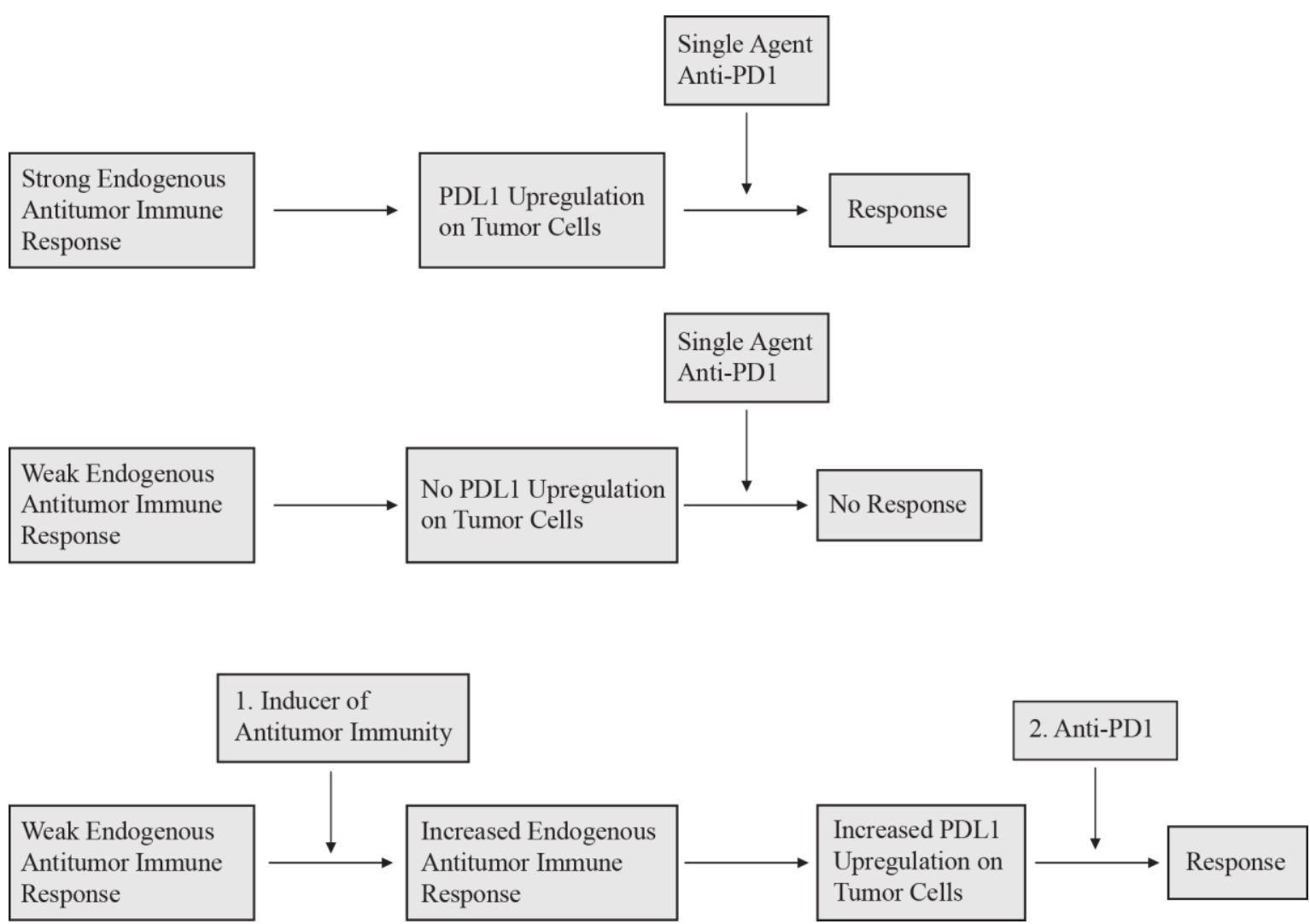

Figure 5. The above figure shows the significance of combination therapies. When a tumor is strongly immunogenic, then even if an antibody against the single immune checkpoint receptor is used as a single agent, we can see a good response. Whereas if the tumor is poorly immungenic then the endogenous immune response will not be able to eliminate the tumor if an antibody against an immune checkpoint receptor is used as a single agent therapy. Therefore an antitumor immunity enhancer must be used in order to boost the endogenous immune response and later an antibody to block the immune checkpoint pathway can be used inorder to see a good response.

\section{OTHER PROMISING IMMUNE CHECKPOINTS AND THEIR CLINICAL STATUS}

Given below in Table 1 are a few immune checkpoint receptors and their ligands. They are currently being studied extensively. The blockade of these immune checkpoints also holds a lot of potential.

Table 1. Few promising immune checkpoint receptors and their ligands

$\begin{array}{ll}\text { Immune Checkpoint Receptor } & \text { Ligand } \\ \text { Lymphocyte Activation Gene 3 (LAG 3) } & \text { MHC class II } \\ \text { B and T Lymphocyte Attenuator (BTLA) } & \text { Herpes Virus Entry Mediator (HVEM) } \\ \text { T cell membrane protein 3 (TIM3) } & \text { Galectin } 9 \\ \text { Adenosine A2a receptor (A2aR) } & \text { Adenosine } \\ \text { Receptor not yet known } & \text { B7-H3 } \\ \text { Receptor not yet known } & \text { B7-H4 }\end{array}$


Preclinical mouse models of cancer have shown that blockade of many of these individual immune checkpoint ligands or receptors can enhance antitumor immunity, and dual blockade of coordinately expressed receptors can produce synergistic antitumor activities. Inhibitors for a number of these immune checkpoint targets are either entering the clinic or are under active development. The clinical status of few of antibodies used to block the immune checkpoint receptors are given below in Table 2.

Table 2. Current clinical status of few antibodies used to block immune checkpoint pathways

$\begin{array}{lll}\text { Target } & \text { Antibody } & \text { Clinical Status } \\ \text { CTLA4 } & \text { Ipilimumab } & \begin{array}{l}\text { US FDA approved for melanoma in 2010. Phase II } \\ \text { and Phase III trials ongoing for multiple cancers. }\end{array} \\ \text { PD1 } & \text { MDX-1106 (BMS-936558) } & \begin{array}{l}\text { Phase I and Phase II clinical trials ongoing in } \\ \text { patients with melanoma, renal and lung cancers. }\end{array} \\ \text { PDL1 } & \text { MDX-1105 } & \begin{array}{l}\text { Phase I clinical trials ongoing in multiple types of } \\ \text { cancers. }\end{array} \\ \text { LAG3 } & \text { IMP321 } & \begin{array}{l}\text { Phase III clinical trials ongoing in patients with } \\ \text { breast cancer. }\end{array} \\ \text { B7-H3 } & \text { MGA271 } & \text { Phase I clinical trials ongoing in multiple cancers. }\end{array}$

\section{IMMUNOSUPPRESSION IN THE TUMOR MICROENVIRONMENT BY THE REGULATORY T CELLS ( $\left.\mathbf{T}_{\mathrm{REG}}\right)$}

$\mathrm{CD} 4+\mathrm{CD} 25+\mathrm{T}_{\mathrm{Reg}}$ cells are crucial for the maintenance of self-tolerance. They play a major role to suppress the effector $\mathrm{T}$ cell functions and reduce the collateral tissue damage during immune responses and hence play a major role in the regulation of autoimmune disorders. $\mathrm{T}_{\text {Reg }}$ cells were found to accumulate in the tumor micro environment and are thought to represent a major immune resistance mechanism that helps the cancer cells to form a sustainable tumor mass. They express the forkhead transcription factor FOXP3. They do not express cell surface molecules that are unique to them, but they do express high levels of multiple immune checkpoint receptors. Genes encoding for some of these immune checkpoint receptors, such as CTLA4, are actually the target genes for the forkhead transcription factor FOXP3 [44, 45]. Although, these immune checkpoint receptors inhibit the effector $\mathrm{T}$ cell functions, they enhance the $\mathrm{T}_{\mathrm{Reg}}$ cell activity or proliferation. An antibody that specifically binds to the $\mathrm{T}_{\mathrm{Reg}}$ cells has not yet been made, many of the monoclonal antibodies currently in clinical testing block the immune checkpoint receptors that are present on the $\mathrm{T}_{\text {Reg }}$ cells, which might reduce the activity or proliferation of the $\mathrm{T}_{\text {Reg }}$ cells, which eventually leads to the enhancement of the effector T cell functions.

\section{CONCLUSION AND FUTURE IMPLICATIONS}

Cancer immunotherapies have made significant strides in the past few years due to the improved understanding of tumor biology and immunology. Therapeutic antibodies currently provide clinical benefit to patients with cancer and have been established as standard care agents for several highly prevalent human cancers. There will be many advances in the field of cancer immunotherapies, over the next decade, that will arise in the form of identification of new targets, manipulation of the immune checkpoints and optimization of the antibody structure to promote the amplification of antitumor immune responses.

\section{ACKNOWLEDGEMENTS}

I would like to thank Arghyadeep Dash, my fellow friend, who has designed the figures used in this review paper. I am also thankful to Dr. Sathish Narayanan and Dr. Manish Pathak for their continued guidance and motivational support. I am grateful to KVPY for funding my education. 


\section{REFERENCES}

[1]. Hathcock, K. S. et al.Identification of an alternative CTLA-4 ligand costimulatory for T cell activation. Science 262,1993,905-907.

[2]. Freeman, G. J.et al.Cloning of B7-2: a CTLA-4 counter-receptor that costimulates human T cell proliferation. Science 262, 1993, 909-911.

[3]. Azuma, M. et al.B70 antigen is a second ligand for CTLA-4 and CD28. Nature 366, 1993, 76-79.

[4]. Linsley, P. S., Clark, E. A. \& Ledbetter, J. A. T-cell antigen CD28 mediates adhesion with B cells by interacting with activation antigen B7/BB-1. PNAS USA 87,1990,5031-5035.

[5]. Linsley,P. S.et al.CTLA-4 is a second receptor for the B cell activation antigen B7.J. Exp.Med. 174,1991,561-569.

[6]. Rudd, C. E., Taylor, A. \& Schneider, H. CD28 and CTLA-4 coreceptor expression and signal transduction. Immunol. Rev. 229, 2009, 12-26.

[7]. Linsley, P. S.et al.Human B7-1 (CD80) and B7-2 (CD86) bind with similar avidities but distinct kinetics to CD28 and CTLA-4 receptors. Immunity 1,1994, 793-801.

[8]. Riley, J. L. et al.Modulation of TCR-induced transcriptional profiles by ligation of CD28, ICOS, and CTLA-4 receptors.PNAS USA 99,2002,11790-11795.

[9]. Schneider, H.et al.Reversal of the TCR stop signal by CTLA-4. Science 313,2006, 1972-1975.

[10]. Egen, J. G. \& Allison, J. P. Cytotoxic T lymphocyte antigen-4 accumulation in the immunological synapse is regulated by TCR signal strength. Immunity $16,2002,23-35$.

[11]. Parry, R. V.et al.CTLA-4 and PD-1 receptors inhibit T-cell activation by distinct mechanisms. Mol. Cell Biol.25,2005, 9543-9553.

[12]. Schneider, H.et al.Cutting edge: CTLA-4 (CD152) differentially regulates mitogen-activated protein kinases (extracellular signal-regulated kinase and c-Jun N-terminal kinase) in CD4+ T cells from receptor/ligand-deficient mice. J. Immunol. 169,2002, 3475-3479.

[13]. Qureshi, O. S.et al.Trans-endocytosis of CD80 and CD86: a molecular basis for the cell-extrinsic function of CTLA-4. Science 332,2011, 600-603.

[14]. Lenschow, D. J., Walunas, T. L. \& Bluestone, J. A. CD28/B7 system of T cell costimulation. Annu. Rev. Immunol. 14, 1996, 233-258.

[15]. Wing, K.et al.CTLA-4 control over Foxp3+ regulatory T cell function. Science 322,2008, 271-275.

[16]. Peggs, K. S., Quezada, S. A., Chambers, C. A., Korman, A. J. \& Allison, J. P. Blockade of CTLA-4 on both effector and regulatory $\mathrm{T}$ cell compartments contributes to the antitumor activity of anti-CTLA-4 antibodies. J. Exp. Med. 206,2009, 1717-1725.

[17]. Tivol, E. A.et al.Loss of CTLA-4 leads to massive lymphoproliferation and fatal multiorgan tissue destruction, revealing a critical negative regulatory role of CTLA-4. Immunity 3,1995, 541-547.

[18]. Waterhouse, P.et al.Lymphoproliferative disorders with early lethality in mice deficient in Ctla-4. Science 270, 1995, 985-988.

[19]. Ribas, A. Clinical development of the anti-CTLA-4 antibody tremelimumab. Semin. Oncol. 37,2010, 450-454

[20]. Hodi,F.S.et al.Improved survival with ipilimumab in patients with metastatic melanoma.NEJM.363,2010,711-723.

[21]. Pardoll, D. M. The blockade of immune checkpoints in cancer immunotherapy. Nature reviews. Cancer, 12(4),2012, 252-64.

[22]. Ishida, Y., Agata, Y., Shibahara, K. \& Honjo, T. Induced expression of PD-1, a novel member of the immunoglobulin gene superfamily, upon programmed cell death. EMBO J. 11,1992, 3887-3895.

[23]. Freeman, G. J. et al.Engagement of the PD-1 immunoinhibitory receptor by a novel B7 family member leads to negative regulation of lymphocyte activation. J. Exp. Med. 192,2000, 1027-1034.

[24]. Keir, M. E. et al.Tissue expression of PD-L1 mediates peripheral T cell tolerance. J. Exp. Med. 203,2006,883-895.

[25]. Nishimura, H. et al.Autoimmune dilated cardiomyopathy in PD-1 receptor-deficient mice.Science 291,2001, 319322.

[26]. Nishimura, H., Nose, M., Hiai, H., Minato, N. \&Honjo, T. Development of lupus-like autoimmune diseases by disruption of the PD-1 gene encoding an ITIM motif-carrying immunoreceptor. Immunity 11,1999,141-151.

[27]. Okazaki, T. \& Honjo, T. PD-1 and PD-1 ligands: from discovery to clinical application. Int. Immunol. 19,2007,813-824

[28]. Keir, M. E., Butte, M. J., Freeman, G. J. \& Sharpe, A. H. PD-1 and its ligands in tolerance and immunity. Annu. Rev. Immunol. 26,2008, 677-704.

[29]. Dong, H. et al.Tumor-associated B7-H1 promotes T-cell apoptosis: a potential mechanism of immune evasion. Nature Med. 8,2002, 793-800.

[30]. Blank, C. et al.PD-L1/B7H-1 inhibits the effector phase of tumor rejection by T cell receptor (TCR) transgenic CD8+ T cells. Cancer Res. 64,2004, 1140-1145.

[31]. Dong, H., Zhu, G., Tamada, K. \& Chen, L. B7-H1, a third member of the B7 family, co-stimulates T-cell proliferation and interleukin-10 secretion. Nature Med. 5,1999, 1365-1369.

[32]. Latchman,Y.et al.PD-L2 is a second ligand for PD-1 and inhibitsT cell activation. Nature Immunol.2,2001,261268.

[33]. Butte, M. J., Keir, M. E., Phamduy, T. B., Sharpe, A. H. \& Freeman, G. J. Programmed death-1 ligand 1 interacts specifically with the B7-1 costimulatory molecule to inhibit T cell responses. Immunity 27,2007,111-122.

[34]. Tseng, S. Y. et al.B7-DC, a new dendritic cell molecule with potent costimulatory properties for T cells. J. Exp. Med. 193,2001, 839-846. 
[35]. Fife, B. T. et al.Interactions between PD-1 and PD-L1 promote tolerance by blocking the TCR-induced stop signal. Nature Immunol. 10,2009, 1185-1192.

[36]. Francisco, L. M. et al.PD-L1 regulates the development, maintenance, and function of induced regulatory T cells. J. Exp. Med. 206,2009, 3015-3029.

[37]. Terme, M. et al.IL-18 induces PD-1-dependent immunosuppression in cancer. Cancer Res. 71,2011,5393-5399.

[38]. Fanoni, D. et al.New monoclonal antibodies against B-cell antigens: possible new strategies for diagnosis of primary cutaneous B-cell lymphomas. Immunol. Lett.134,2011, 157-160.

[39]. Velu, V. et al.Enhancing SIV-specific immunity in vivo by PD-1 blockade. Nature 458,2009, 206-210.

[40]. Paterson, A. M. et al.The programmed death-1 ligand 1:B7-1 pathway restrains diabetogenic effector T cells in vivo. J. Immunol. 187,2011, 1097-1105.

[41]. Park, J. J. et al.B7-H1/CD80 interaction is required for the induction and maintenance of peripheral T-cell tolerance. Blood 116,2010, 1291-1298.

[42]. Sznol, M. et al. Safety and antitumor activity of biweekly MDX 1106 (Anti PD 1) in patients with advanced refractory malignancies. J. Clin. Oncol. 28 (Suppl.),2010,Abstract 2506.

[43]. McDermott, D. F. et al. A phase I study to evaluate safety and antitumor activity of biweekly MDX 1106 (Anti PD 1)in patients with RCC and other advanced refractory malignancies.J.Clin. Oncol. 29(Suppl. 7),2011,Abstract 331.

[44]. Hori, S., Nomura, T. \& Sakaguchi, S. Control of regulatory T cell development by the transcription factor Foxp3. Science 299,2003, 1057-1061.

[45]. Fontenot, J. D., Gavin, M. A. \& Rudensky, A. Y. Foxp3 programs the development and function of CD4+ CD25+ regulatory T cells. Nature Immunol. 4,2003,330-336. 